\title{
Dust in the Intergalactic Medium of Galaxy Clusters
}

\author{
M. Stickel \\ Max-Planck-Institut für Astronomie, Königstuhl 17, D-69117 \\ Heidelberg, Germany
}

\begin{abstract}
Far-infrared (FIR) observations with ISOPHOT aboard the Infrared Space Observatory were carried out to observe the thermal FIR emission from diffuse intracluster dust (ICD) in galaxy clusters and outlying dust structures in the elliptical galaxies M86 and NGC 5128 (Centaurus A). Extended FIR emission from ICD has only been detected in the Coma cluster. It is likely caused by tidal interactions, since Coma is a dynamically young cluster with on-going gravitational interactions in the centre and infalling galaxy groups. M86 has a complex FIR morphology with a compact double source near its centre and several off-centre sources. The brightest FIR source lies between M86 and the nearby spiral NGC 4402 and represents the first direct detection of a localized intracluster dust cloud not associated with optical or neutral hydrogen emission. Overall, the FIR data do not support the ram pressure dust stripping scenario suggested on the basis of IRAS data, but indicate a significant influence of tidal interactions. NGC 5128 shows obvious optical signs of interaction, most notably a central dust lane and stellar shells. The FIR emission of outlying dust $15 \mathrm{kpc}$ from the centre has been discovered where previously $\mathrm{HI}$ and molecular gas closely associated with the shells had been found. NGC 5128 is the first case of a shell galaxy where all components of the interstellar medium (ISM) are detected far off the nucleus, indicating an inner disk origin of the outlying material.
\end{abstract}

\section{Introduction}

The evolution of galaxies is influenced by several dynamical interactions with other galaxies and the surrounding medium. Gravitational interactions, tidal stripping, and merging severely affect and rearrange the stellar as well as the gaseous content, as indicated by disturbed morphologies observed in the optical and HI (Hibbard et al. 2001). Ram pressure stripping removes at least the outer HI envelope, leaving significantly truncated HI disks. Outflows driven by internal processes related to the star formation history can replenish the ISM lost from the halo region.

Dust as a component of the ISM mixed with the interstellar atomic and molecular gas is expected to be entrained in outflowing winds, reaching large heights above spiral disks, and to participate in both ram and tidal stripping processes, transferring it to the outer regions and even into the intergalactic space. However, observational evidence for both the dust transfer processes as 

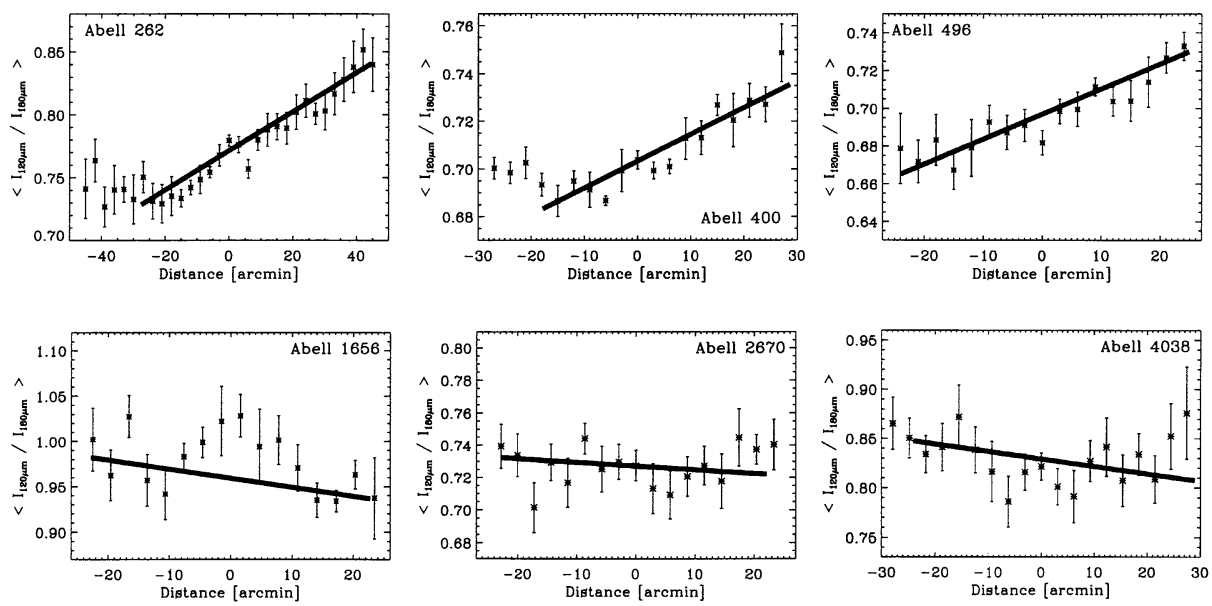

Figure 1. Zodiacal light subtracted $\mathrm{I}_{120 \mu \mathrm{m}} / \mathrm{I}_{180 \mu \mathrm{m}}$ profiles as a function of distance from the centre for the six clusters. Within the errors, a straight line is good representation of the color profiles for five out of six clusters. Only Abell 1656 (Coma) shows a significant bump in the centre, indicating the presence of FIR emitting material.

well as the presence of dust distributed far away from galaxy centres and in the intergalactic region is still scarce.

Diffuse ICD extended on scales of a cluster core radius and compact dust structures in the outer regions of galaxies can give rise to optical depth effects such as absorption and reddening. These indirect dust tracers, however, are notoriously difficult to detect. A more promising way to localize dust far away from galaxy centres and between galaxies is the direct detection of its thermal FIR emission. The distribution of extended dust removed or expelled from galaxies likely follows roughly the galaxy density distribution. Any extended emission of a diffuse dust component distributed between galaxies is thus most easily detected in groups and clusters, where the galaxy density is high and tidal stripping by nearby encounters is frequent. Compact dust clouds resulting from tidal interactions can most easily be detected as compact FIR emission regions in the outer stellar halos of elliptical galaxies. While dust is mostly confined to the narrow disk in spiral galaxies, dust being transferred to the intergalactic region by winds can be recognized as thick FIR disks in edge-on spiral galaxies.

The diffuse FIR emission on scales of a cluster core radius in the central regions of six galaxy clusters (Stickel et al. 1998, 2002) and compact dust structures in the outer regions of the cluster elliptical galaxies M86 (Stickel et al. 2003a) and NGC 5128 (Stickel et al. 2003b) were detected with the ISOPHOT detector (Lemke et al. 1996) aboard the Infrared Space Observatory (Kessler et al. 1996). It provided higher sensitivity, higher angular resolution, and an increased wavelength coverage compared to IRAS, which is particularly crucial for detection of very cold dust in the outer regions of galaxies, where the dust in only heated by the diffuse star light. 


\section{Diffuse Intracluster Dust}

The extended diffuse FIR emission from ICD was investigated in a small sample of six galaxy clusters. The observed clusters cover a range of parameters such as distance, X-ray morphology, X-ray temperature, and inferred cooling flow rate. Crossed scans at $120 \mu \mathrm{m}$ and $180 \mu \mathrm{m}$ allow the derivation of $\mathrm{I}_{120 \mu \mathrm{m}} / \mathrm{I}_{180 \mu \mathrm{m}}$ color profiles, where FIR emitting material should give rise to a dip or bump, depending on its temperature. The sample included Abell 1656 (Coma), where optical studies had found evidence for ICD (Karachentsev \& Lipovetskii 1969), and Abell 262 and Abell 2670, where extended FIR emission was detected on IRAS plates (Wise et al. 1993).

The interpretation of the $\mathrm{I}_{120 \mu \mathrm{m}} / \mathrm{I}_{180 \mu \mathrm{m}}$ color profiles is hampered by the presence of galactic cirrus emission from the Milky Way, which also leads to a dip or bump and thus can mimic ICD. In fact, three out of six clusters (Abell 262, Abell 1656, Abell 2670) did show a systematic change in the raw $\mathrm{I}_{120 \mu \mathrm{m}} / \mathrm{I}_{180 \mu \mathrm{m}}$ color profiles. Zodiacal light subtraction breaks the degeneracy between ICD and foreground galactic cirrus (Stickel et al. 2002), and only Abell 1656 (Coma) shows a bump in the zodiacal light subtracted $\mathrm{I}_{120 \mu \mathrm{m}} / \mathrm{I}_{180 \mu \mathrm{m}}$ color profile (Fig. 1) This indicates that the FIR emission seen on IRAS plates in the direction of Abell 262 and Abell 2670 is in fact foreground galactic cirrus, while the extended FIR emission in Abell 1656 (Coma) is interpreted as thermal emission from ICD.

The total inferred dust mass for Abell 1656 is low $\left(\mathrm{M}_{\mathrm{D}} \approx 10^{7} \mathrm{M}_{\odot}\right)$ and the associated visual extinction negligible $\left(A_{V} \ll 0.1 \mathrm{mag}\right)$. This together with the absence of any evidence for ICD in the other five observed clusters indicates that ICD is likely not responsible for the excess X-ray absorption seen in cooling flow clusters, which in turn represents another unsuccessful attempt in detecting the presumed final stage of the cooling flow material. Furthermore, the observed dimming of high-redshift supernovae is unlikely to be attributable to extinction caused by dust in the intracluster or even a presumed intercluster medium. The likely cause for the dust in the intracluster medium (ICM) of Abell 1656 are tidal interactions, since it is a dynamically young cluster with on-going gravitational interactions and infalling galaxy groups.

\section{Localized Intracluster Dust}

\subsection{The Virgo elliptical M86}

M86 is a candidate for ram pressure stripping of its surrounding hot X-ray emitting gas while infalling into the Virgo cluster (Rangarajan et al. 1995). An off-centre FIR source detected by IRAS and closely coincident with extended Xray emission was interpreted as dust removed from M86 by ram pressure stripping, while an asymmetry in the optical isophotes might be caused by starlight scattered by the stripped dust (White et al. 1991).

The FIR morphology of M86 (Fig. 2) is complex and shows a total of seven distinct sources within the optical stellar halo of the elliptical galaxy (Stickel et al. 2003), which is quite unexpected since elliptical galaxies are supposed to have only little dust. The western component of the central double source is closely aligned with the centre of M86 and identified with the FIR emission from the inner part of the M86 galaxy, while the eastern component is likely 

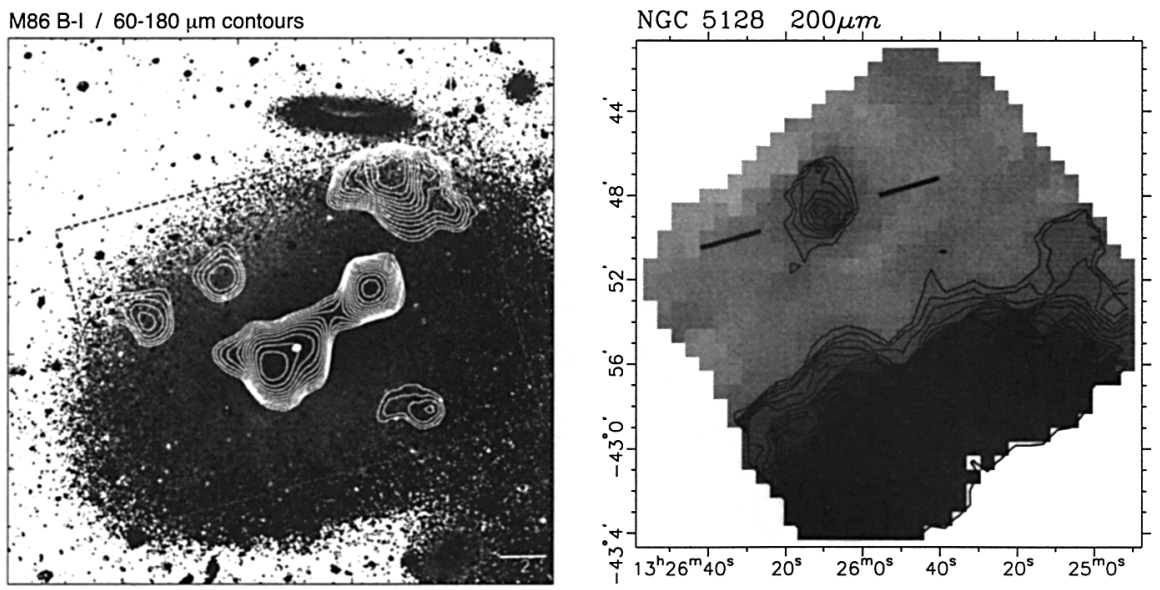

Figure 2. Left: FIR "white light" $(60-180 \mu \mathrm{m})$ contours of M86 overlaid on a gray-scale representation of the optical B-I color index image. The complex FIR morphology consists of a total of seven resolved and unresolved components. Most notably is the core-halo source between M86 and NGC 4402 (upper right corner of FIR mapped region). Right: Gray-scale representation with overlaid contours of the $200 \mu \mathrm{m}$ map of the region north of NGC 5128. In addition to the strong FIR emission of the central dust disk of NGC 5128 (bottom), there is an extended emission region $\approx 15^{\prime}$ north-east (marked with a bar).

associated with dust streamers already discovered in the optical. Both have a cold FIR spectral energy distribution (SED) with $\mathrm{T}_{\text {Dust }} \approx 18 \mathrm{~K}$. The previously detected off-centre FIR source $\approx 3.5^{\prime}$ north-west is unresolved and positionally coincident with an unresolved optical counterpart. It has in contrast a rather warm FIR SED with a dust temperature of $\mathrm{T} z 60 \mathrm{~K}$. It lies away from the extended X-ray emission, and is likely a background object, not ram pressure stripped dust.

A particularly interesting FIR source lies between M86 and the nearby spiral NGC 4402. It has a core-halo structure, no obvious optical identification, and is not associated with $\mathrm{HI}$ gas, but lies at the position of the strong northern decline in X-ray brightness. It has a rather large dust mass of $\approx 1.5 \times 10^{7} M_{\odot}$ and is most likely interpreted as ISM tidally removed from NGC 4402. It represents the first direct detection of a localized intracluster dust cloud.

\subsection{The disturbed shell elliptical NGC 5128 (Centaurus A)}

The elliptical NGC 5128 (Centaurus A) is an optically highly disturbed system with a central dust lane, resulting from at least one major merger with a spiral galaxy. It has a system of surrounding optical shells, and HI was detected in several regions near the outer shells (Schiminovich et al. 1994). Molecular gas traced by $\mathrm{CO}$ emission found in the two regions with the strongest $\mathrm{HI}$ emission (Charmandaris et al. 2000) indicated that the ISM in the tidal remnant has not yet completely settled in the centre of the potential. 
A somewhat extended FIR emission region is present in both the $150 \mu \mathrm{m}$ and $200 \mu \mathrm{m}$ maps (Fig. 2) in the northern shell region (Stickel et al. 2003b), where previously neutral hydrogen and molecular gas has been found. The FIR SED can be reconciled with a modified blackbody spectrum with a very cold dust color temperature of $\mathrm{T}_{\text {Dust }} \approx 13 \mathrm{~K}$. A representative value for the associated dust mass is $\mathrm{M}_{\text {Dust }} \approx 7 \times 10^{4} \mathrm{M}_{\odot}$, which together with the HI gas mass gives a gas-to-dust ratio of $\approx 300$, close to that of normal inactive spiral galaxies. This together with the normal ratio of atomic to molecular gas mass indicates that the interstellar medium from the inner part of a captured disk galaxy is likely the origin of the outlying gas and dust. This is the first case where all three components (atomic gas, molecular gas, dust) of the ISM of a captured galaxy has been detected in the outskirts of a shell galaxy.

These observations are in agreement with recent theoretical considerations that in galaxy interactions leading to stellar shell structures the less dissipative clumpy component of the ISM from the captured galaxy can lead to gaseous shells. Alternatively, the outlying gas and dust in NGC 5128 could be a rotating ring structure resulting from an interaction or even late infall of tidal material of a merger in the distant past.

\section{Concluding Remarks}

In all the above described cases with dust far off from the galaxy centres, there is strong evidence for gravitational interactions going on between the involved galaxies or galaxy groups. Tidal interaction thus appears to be a important process of dust removal from cluster galaxies and its transfer to the intergalactic space, and for the origin of outlying dust structures in cluster ellipticals.

Therefore, interacting galaxies within compact galaxy groups should also show evidence of extended intergalactic dust. A prime candidate for such a group is Stephan's Quintet (HCG 92), where allmost all HI as well as molecular gas with an accompanying star forming region is found in the intergalactic space (Xu et al. 1999). No evidence for extended FIR emission not associated with the galaxies is found in the IRAS data (Allam et al. 1996), but the ISOPHOT $100 \mu \mathrm{m}$ clearly shows a diffuse component surrounding the individually detected galaxies (Fig. 3). The comparison with the $60 \mu \mathrm{m}$ data, where this diffuse component is absent, indicates that the dust in the intergalactic space is rather cold with a SED increasing towards longer FIR wavelengths.

Eventually, gravitational interaction may even play an important role in redistributing the dust in inconspicuous, apparently undisturbed galaxies. A few spiral galaxies seen edge-on are known to have dust at high $\mathrm{z}$ - heights above their disks. A particularly notable example is the extended FIR emission along the minor axis of the star-burst galaxy NGC 253. This thick disk of FIR emission appears to be indicative of a dust distribution being more extended than the stellar light and is attributed to a large scale outflow (Radovich et al. 2001). However, a faint stellar halo has been detected in a deep optical image (Malin \& Hadley 1997), which closely resembles the thick dust disk. Again, the origin for the diffuse star light is a gravitational encounter or merging (Malin \& Hadley 1997), which in turn is also a viable but as yet disregarded explanation for the extended FIR emission from dust. 

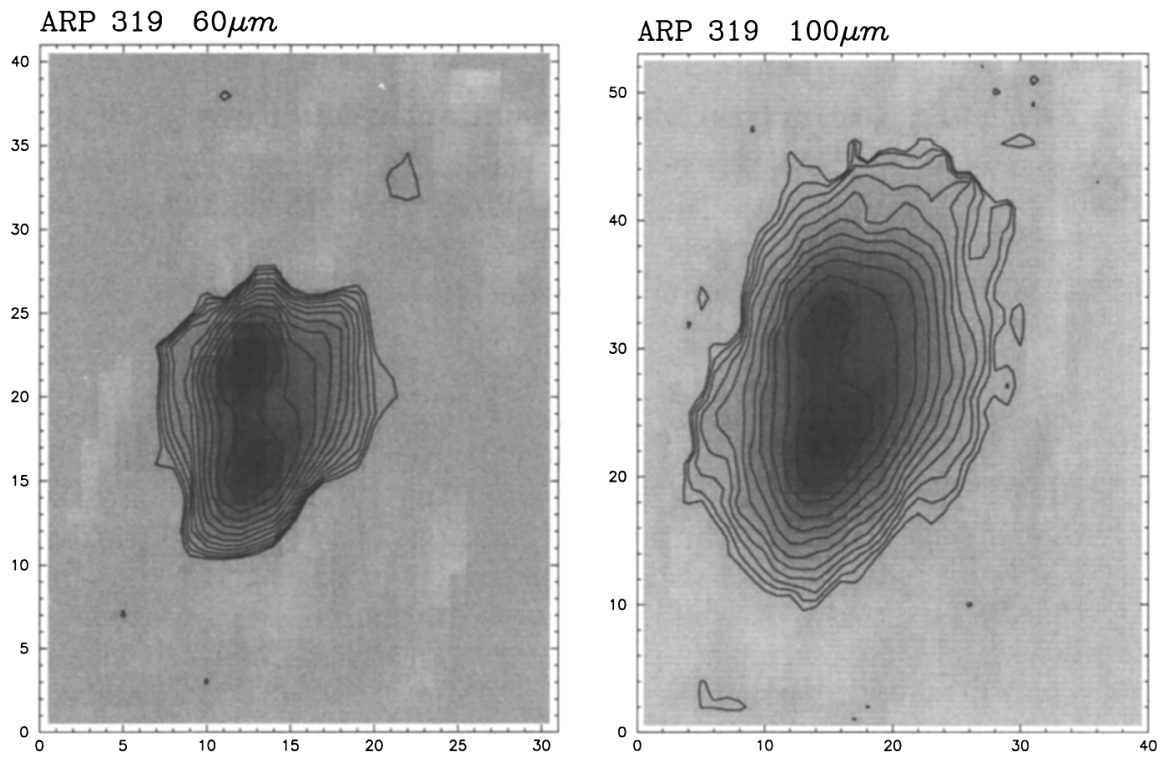

Figure 3. Gray-scale representation with overlaid contours of the $60 \mu \mathrm{m}$ (left) and $100 \mu \mathrm{m}$ (right) ISOPHOT maps of Stephan's Quintet (HCG 92). The galaxy group at $100 \mu \mathrm{m}$ is embedded in a diffuse envelope surrounding the interacting galaxies, indicating an extended distribution of cold dust.

\section{References}

Allam S., et al. 1996, A\&AS, 117, 39

Charmandaris V., Combes F., van der Hulst J.M. 2000, A\&A, 356, L1

Hibbard J.E., et al. 2001, ASP Conf. Ser. 240, 657

Karachentsev I.D., Lipovetskii V.A. 1969, Soviet Phys., 12, 909

Kessler M.F., et al. 1996, A\&A, 315, L27

Lemke D., et al. 1996, A\&A, 315, L64

Malin D., Hadley B. 1997, PASA, 14, 52

Radovich M., Kahanpää J., Lemke D., 2001, A\&A, 377, 73

Rangarajan F.V.N., et al. 1995, MNRAS, 277, 1047

Schiminovich D., et al. 1994, ApJ, 423, L101

Stickel M., et al. 1998, A\&A, 329, 55

Stickel M., et al. 2002, A\&A, 383, 367

Stickel M., et al. 2003a, A\&A, 397, 503

Stickel M., et al. 2003b, A\&A, in press

White D.A., et al. 1991, ApJ, 375, 35

Wise M.W., et al. 1993, ApJ, 405, 94

Xu C., Sulentic J., Tuffs, R. 1999, ApJ, 512, 178 